\title{
Percepção dos pais de crianças com estomia intestinal a respeito das orientações de enfermagem
}

Perception of the parents of children with intestinal stoma regarding the nursing orientations

Percepción de los padres de niños con estomia intestinal respecto de las orientaciones de enfermería

Elisa Maria Bezerra Maia ${ }^{1, *}$, Gisela Maria Assis ${ }^{1}$

ORCID IDS

Maia EMB (iD https://orcid.org/0000-0002-1768-1928

Assis GM (D) https://orcid.org/0000-0001-6343-8075

\section{COMO CITAR}

Maia EMB, Assis GM. Percepção dos pais de crianças com estomia intestinal a respeito das orientações de enfermagem. ESTIMA, Braz. J. Enterostomal Ther., 17: e0819. https://doi.org/10.30886/estima. v17.663 PT

\section{RESUMO}

Objetivo: O presente estudo teve o objetivo de identificar a percepção dos pais de crianças com estomia de eliminação intestinal acerca das orientações recebidas pela equipe de saúde durante a hospitalização para sua confecção. Métodos: Trata-se de estudo transversal, qualiquantitativo. A coleta de dados ocorreu na enfermaria de cirurgia pediátrica de um hospital universitário por meio de entrevista semiestruturada, gravada e composta por perguntas abertas e fechadas. Resultados: Participaram da pesquisa nove pais de crianças com estomia de eliminação intestinal em acompanhamento pela equipe de cirurgia pediátrica. Posteriormente, foram submetidos à análise estatística simples e categorial temática de Bardin. Com relação às principais complicações, a presença de dermatite foi apontada por $100 \%$ da amostra e as infecções fúngicas seguidas de prolapso por 44,4\%. O estudo demonstrou que a falta de conhecimento resulta em complicações e insegurança. A rede de apoio constitui um dos recursos utilizados para a busca de informação. Um manual com orientações foi apontado como estratégia para o cuidado no domicílio. Conclusão: Apesar das falhas na orientação e do apoio aos pais de crianças com estomia, os feedbacks para auxílio nas mudanças e sugestões de estratégias válidas foram importantes.

DESCRITORES: Educação em saúde; Colostomia; Enfermagem; Estomaterapia.

1.Universidade Federal do Paraná - Complexo Hospital de Clínicas - Comissão de Cuidados com a Pele - Curitiba/PR - Brasil

*Autor correspondente: elisamaia13@hotmail.com

Recebido: Nov. 032018 | Aceito: Maio 022019 


\begin{abstract}
Objective: The present study aimed to identify the perception of the parents of children with intestinal elimination stoma about the guidelines received by the health team during hospitalization for their preparation. Methods: This is a cross-sectional, qualitative and quantitative study. Data collector occurred in the pediatric surgery ward of a university hospital through a semi-structured interview, recorded and composed of open and closed questions. Results: Nine parents of children with intestinal elimination stoma were followed up by the pediatric surgery team. Subsequently, it was submitted to the simple and categorical statistical analysis of Bardin. Regarding the main complications, the presence of dermatitis was indicated by $100 \%$ of the sample and the fungal infections followed by prolapse by 44.4\%. The study showed that the lack of knowledge results in complications and insecurity. The support network is one of the resources used to search for information. A manual with guidelines was pointed out as a strategy for home care. Conclusion: Despite the failures in the guiding and supporting parents of children with a stoma, the feedbacks to help with changes and suggestions for valid strategies were important.
\end{abstract}

DESCRIPTORS: Health education; Colostomy; Nursing; Stomatherapy.

\title{
RESUMEN
}

Objetivo: El presente estudio tuvo el objetivo de identificar la percepción de los padres de niños con estomia de eliminación intestinal acerca de las orientaciones recibidas por el equipo de salud durante la hospitalización para su confección. Métodos: Se trata de un estudio transversal, cualiquantitativo. La recolección de datos ocurrió en la enfermería de cirugía pediátrica de un hospital universitario por medio de entrevista semiestructurada, grabada y compuesta por preguntas abiertas y cerradas. Resultados: Participaron de la investigación nueve padres de niños con estomia de eliminación intestinal en seguimiento por el equipo de cirugía pediátrica. Posteriormente, fueron sometidos al análisis estadístico simple y categorial temático de Bardin. Con respecto a las principales complicaciones, la presencia de dermatitis fue apuntada por el 100\% de la muestra y las infecciones fúngicas seguidas de prolapso por el 44,4\%. El estudio demostró que la falta de conocimiento resulta en complicaciones e inseguridad. La red de apoyo constituye uno de los recursos utilizados para la búsqueda de información. Un manual con orientaciones fue apuntado como estrategia para el cuidado en el domicilio. Conclusión: A pesar de las fallas en la orientación y el apoyo a los padres de niños con estomia, los feedbacks para ayudar en los cambios y sugerencias de estrategias válidas fueron importantes.

DESCRIPTORES: Educación en salud; Colostomía; Enfermería; Estomaterapia.

\section{INTRODUÇÃO}

Considera-se a estomia uma intervenção cirúrgica com a função de facilitar a comunicação entre um órgão interno e o meio externo ${ }^{1}$. Quando em áreas intestinais, podem ser confeccionadas tanto no intestino delgado (ileostomia) quanto no grosso (colostomia), o qual se conecta à parede abdominal por meio de uma incisão cirúrgica com intuito de permitir o esvaziamento intestinal ${ }^{2}$.

A presença de uma estomia pode gerar sequelas e déficits no estado geral de saúde, fazendo-se necessários cuidados contínuos. Quando essa condição se dá na infância, os cuidados podem vir a ser mais intensos, considerando as angústias e medos da própria criança e de seus familiares ${ }^{3}$.

As principais indicações para colostomias na infância são as anomalias anorretais, megacólon congênito, atresias colônicas e retais, doenças inflamatórias, traumatismos perineais, enterocolite necrotizante e infecções pelviperineais com a finalidade de descompressão, proteção e evacuação ${ }^{2}$.

A confecção adequada de uma estomia é essencial para proporcionar qualidade de vida $(\mathrm{QV})$ à criança ${ }^{4}$. Além disso, os cuidados terapêuticos que envolvem essas crianças devem ser orientados e guiados por uma equipe multidisciplinar para que a família se sinta mais segura em lidar com os desafios que tal condição proporciona ${ }^{1,3,5}$.

Para isso, as orientações devem ser esclarecedoras quanto à definição de estomias, para que servem, como manusear e como será a rotina de cuidados diários a essa criança, sempre trazendo os aspectos positivos e os benefícios ${ }^{1,5}$. É importante permitir que a criança e sua família manipulem os materiais que farão uso para prevenir surpresas no pós-operatório imediato ${ }^{3}$.

Há escassez de referências que indiquem orientações específicas sobre a alta da criança com estomia ${ }^{3}$. Considerando-se que compete ao enfermeiro interagir e planejar com a equipe multidisciplinar e a família na condução das orientações para alta, desde o momento da internação para confecção da estomia até a alta da criança para o domicilio ${ }^{5}$, espera-se que esse profissional atue também no desenvolvimento de material científico de qualidade nessa temática. 
Algumas das complicações da estomização podem ser a formação de hérnia, infecções locais, retrações, estenoses, prolapsos e obstrução intestinal ${ }^{6}$. O cuidado para se evitar alguns desses problemas inclui a limpeza local, o uso adequado de bolsas coletoras, o controle dietético para evitar a alteração do $\mathrm{pH}$ das fezes e o tratamento das dermatites periestomais e das infecções secundárias locais² .

Tendo em vista as dificuldades de aceitação e de cuidado da criança com estomia, apontadas como consequência das lacunas do planejamento da assistência de enfermagem e da orientação durante o processo operatório, é necessária a ampliação das investigações relacionadas à experiência dos pais sobre a temática, com intuito de que esses desenvolvam habilidades para melhora no cuidado domiciliar.

Este estudo teve por objetivo identificar a percepção dos pais de crianças com estomia de eliminação intestinal acerca das orientações recebidas durante hospitalização para sua confecção.

\section{MÉTODOS}

Trata-se de estudo transversal de caráter exploratório e descritivo com abordagem qualiquantitativa realizado em um hospital universitário da região sul do Brasil. O estudo foi aprovado pelo Comitê de Ética em Pesquisa com Seres Humanos sob parecer 2062061/2017.

Para participar da pesquisa, selecionou-se a amostra composta por pais de crianças com idade entre zero e 11 anos, com estomia de eliminação intestinal confeccionada entre janeiro de 2014 e dezembro de 2016, que estiveram em acompanhamento ambulatorial pelo hospital e que receberam cuidado direto e domiciliar realizado pelos pais. Os dados foram coletados entre julho e dezembro de 2017, por meio de entrevistas audiogravadas e realizadas no momento anterior ou posterior às consultas de acompanhamento em ambulatório da equipe de cirurgia pediátrica. Utilizou-se instrumento semiestruturado, construído pelas pesquisadoras, com base na literatura consultada. Para caracterização da amostra, investigaram-se as variáveis sexo, idade, diagnóstico clínico, tipos de estomia, orientações recebidas pela equipe multiprofissional no período pré- e pós-operatório, complicações e demarcação do local da estomia. Para a etapa qualitativa, utilizaram-se as seguintes questões: "Como foi a experiência de cuidar de seu filho com estomia?" e "O que o hospital poderia fazer para adaptação dos pais de crianças com estomia?”. Os dados quantitativos foram submetidos à análise estatística simples e os qualitativos foram analisados pela técnica de análise categorial temática de Bardin operacionalizada pelas etapas analíticas: pré-análise; exploração do material; e tratamento dos resultados obtidos e interpretação ${ }^{7}$.

$\mathrm{Na}$ etapa pré-análise, a pesquisadora fez a transcrição na íntegra das entrevistas, a leitura flutuante para apreender as principais ideias contidas e a elaboração dos indicadores dos fragmentos de texto relacionados ao objeto de estudo. $\mathrm{Na}$ exploração do material, os dados foram codificados e agrupados em categorias temáticas, buscando as conexões e integrações entre os dados brutos. Por fim, na etapa do tratamento dos dados obtidos e análise, realizaram-se as inferências e as interpretações, norteando-se nos significados e conhecimentos das mensagens emitidas pelos participantes.

Para identificação dos participantes, adotou-se a letra "P" seguida de números arábicos de um a nove (P1, P2, $\mathrm{P} 3$...) correspondentes à ordem cronológica dos encontros.

\section{RESULTADOS E DISCUSSÃO}

Foram entrevistados pais de nove crianças, dentre eles quatro mães, dois pais e três casais mãe e pai. Conforme a Tabela 1, houve maior prevalência de crianças do sexo masculino de até dois anos de idade com diagnóstico clínico, em sua maioria, relacionado a anomalias ou malformações congênitas, com colostomia terminal.

A respeito das orientações recebidas pelos pais, sete participantes relataram receber orientações a respeito da técnica cirúrgica antes da realização da cirurgia e outros dois não foram esclarecidos sobre o procedimento antes da confecção da estomia. Após a cirurgia, todos os pais foram orientados a respeito do procedimento cirúrgico.

Com relação ao número de pais orientados no cuidado direto à estomia, observou-se que $88 \%$ da amostra não foi informada sobre os equipamentos coletores e adjuvantes existentes no mercado e utilizados como recurso para coleta do efluente e tratamento da estomia, $44,4 \%$ não receberam orientação quanto ao recorte para adaptação do equipamento coletor à criança (frequência de troca do equipamento coletor bolsa) e 33,3\% quanto ao esvaziamento e higienização. Outro ponto importante que se apresentou evidente como falha nas orientações (88,8\%) foram as atividades permitidas às crianças que têm algum tipo de estomia de eliminação intestinal, tendo em vista a possibilidade de complicações graves relacionadas (Tabela 2). 
Tabela 1. Caracterização dos filhos dos participantes do estudo quanto a sexo, idade, diagnóstico clínico e tipo de estomia. Curitiba, estado do Paraná, 2018.

\begin{tabular}{|c|c|}
\hline Características & n (\%) \\
\hline \multicolumn{2}{|l|}{ Sexo } \\
\hline Feminino & $3(33,3)$ \\
\hline Masculino & $6(66,7)$ \\
\hline \multicolumn{2}{|l|}{ Idade (anos) } \\
\hline$<1$ & $3(33,3)$ \\
\hline 1 & $2(22,2)$ \\
\hline 2 & $2(22,2)$ \\
\hline 3 & $1(11,1)$ \\
\hline 11 & $1(11,1)$ \\
\hline \multicolumn{2}{|l|}{ Diagnóstico clínico } \\
\hline $\begin{array}{l}\text { P77 Enterocolite necrotizante do feto e do } \\
\text { recém-nascido }\end{array}$ & $1(11,1)$ \\
\hline Q42.3 Imperfuração anal & $2(22,2)$ \\
\hline $\begin{array}{l}\text { Q42.8 Atresia e estenose congênita de outras } \\
\text { partes do cólon }\end{array}$ & $2(22,2)$ \\
\hline Q43.1 Megacólon congênito & $1(11,1)$ \\
\hline Q43.7 Persistência de cloaca & $1(11,1)$ \\
\hline $\begin{array}{l}\text { T81.2 Perfuração e laceração acidentais } \\
\text { durante procedimento não classificado em } \\
\text { outra parte (intestino) }\end{array}$ & $2(22,2)$ \\
\hline \multicolumn{2}{|l|}{ Tipo de estomia } \\
\hline Colostomia terminal & $5(55,6)$ \\
\hline Colostomia em dupla boca & $2(22,2)$ \\
\hline lleostomia & $2(22,2)$ \\
\hline
\end{tabular}

NaTabela 2 também se observa que as principais complicações com a estomia e pele periestomal apontadas foram dermatite (100\%), infecções fúngicas $(44,4 \%)$ e prolapso (44,4\%). Em estudo que verificou a ocorrência de complicações relacionadas à confecção da estomia, notou-se que 15,25\% apresentaram complicações, sendo $44,45 \%$ estenoses, $44,45 \%$ prolapsos e $11,11 \%$ dermatite periestoma ${ }^{8}$. Observou-se grande diferença entre os dois estudos, principalmente em relação ao número de dermatites, o que leva à reflexão dos fatores determinantes dessa realidade. Tal complicação pode estar diretamente relacionada à falha nas orientações sobre o cuidado com a estomia, gerar insegurança e motivar a busca por informação em fontes não seguras, fato apontado pelos participantes deste estudo.

Todos os pais relataram ser a primeira vez que tinham contato com estomia de eliminação intestinal e tal dado reforça a necessidade de terem sido orientados e esclarecidos sobre o processo, para que, além de participarem ativamente, pudessem acompanhar e reivindicar ações em todas as etapas da confecção.
Tabela 2. Orientações recebidas pelos pais e complicações estomais e periestomais das crianças atendidas em um hospital universitário da região sul do Brasil. Curitiba, estado do Paraná, 2017.

\begin{tabular}{|c|c|}
\hline Variáveis & $\mathrm{n}(\%)$ \\
\hline \multicolumn{2}{|l|}{ Orientações pré- e pós-cirúrgicas } \\
\hline Técnica cirúrgica & $9(100)$ \\
\hline Equipamentos e adjuvantes & $2(22,2)$ \\
\hline Avaliação da estomia e pele periestomal & $7(77,8)$ \\
\hline Recorte da bolsa coletora & $5(55,6)$ \\
\hline Frequência de troca da bolsa coletora & $6(66,7)$ \\
\hline Esvaziamento da bolsa coletora & $5(55,6)$ \\
\hline Atividades permitidas e contraindicadas & $2(22,2)$ \\
\hline \multicolumn{2}{|l|}{ Complicações com estoma e pele periestomal } \\
\hline Dermatite & $9(100)$ \\
\hline Infecções fúngicas & $4(44,4)$ \\
\hline Prolapso & $4(44,4)$ \\
\hline Retração & $1(11,1)$ \\
\hline Oclusão & $1(11,1)$ \\
\hline Deiscência mucocutânea & $1(11,1)$ \\
\hline Isquemia & $1(11,1)$ \\
\hline Hérnia & $2(22,2)$ \\
\hline
\end{tabular}

O enfermeiro estomaterapeuta ou capacitado pode auxiliar na etapa de demarcação do local da estomia,indicando,juntamente com a família, o melhor local para essa ser exteriorizada na parede abdominal. A demarcação pode ocorrer no dia anterior ou pouco antes do procedimento cirúrgico, delimitando a região adequada a melhor adaptação do equipamento coletor com vistas ao conforto, melhor convívio familiar e social, com execução das atividades diárias sem prejuízos? .

A seguir serão apresentadas as categorias temáticas resultantes da análise das entrevistas com os participantes, sendo essas: a falta de conhecimento pode resultar em complicações com a estomia e pele periestomal; o desconhecimento causa insegurança e medo para cuidar da criança com estomia no domicílio; o papel da rede de apoio no cuidado domiciliar da criança com estomia; conhecimento gera segurança para cuidar da criança com estomia; estratégias que poderiam contribuir para o cuidado da criança com estomia no domicílio.

\section{A falta de conhecimento pode resultar em complicações com a estomia e pele periestomal}

Os participantes externaram não terem recebido orientações suficientes para cuidar da criança com estomia 
no domicílio, nem mesmo como prevenir complicações graves, como dermatites e prolapso:

"Ele fez prolapso e dermatite fúngica e eu não recebi nenhuma orientação." (P6)

"Mas agora ela fica grande e roxa (a estomia), às vezes fica meio preta, eu nunca tive orientação [...] ninguém me falou que poderia acontecer e que sangra tão fácil.” (P8)

"Está aberta ali a bolsa (pele periestomal), aí vai machucando a pele dele, vai ficando aquele vermelhão [...] em casa eu cuido, nem trago mais aqui (hospital) porque venho e passam a mesma coisa que passo em casa." (P3)

Conforme observado, a maioria dos pais disse não ter sido orientada corretamente a respeito do cuidado direto com a estomia, o que pode ter ocasionado ao menos uma complicação estomal ou periestomal do presente estudo. Nesse sentido, outros autores relatam que a falta de informações sobre a estomia acarretou complicações seguidas de queixas sobre a gestão dos cuidados de saúde e o conhecimento incipiente na temática por parte dos profissionais do serviço, o que levou a condutas indevidas e suas consequências, como o uso de equipamento coletor inadequado, maceração da pele periestomal, irritações e vermelhidão ${ }^{10}$.

Como agravante, percebe-se, no cenário atual, que publicações científicas que abordem o cenário pediátrico da vivência com estomia intestinal são escassas. No entanto, uma revisão integrativa realizada com adultos a respeito de aspectos psicológicos de pacientes com estomias intestinais apresentou a importância da orientação pré-operatória, para diminuição de complicações pós-operatórias, contribuindo para a adaptação e enfrentamento da estomização ${ }^{11}$.

\section{O desconhecimento causa insegurança e medo para cuidar da criança com estomia no domicílio}

Muitos participantes referiram sentimento de insegurança e medo para realizar o cuidado direto à estomia. Destacaram que o fato de não terem qualquer conhecimento prévio sobre o assunto os levou a se sentirem despreparados e incapacitados diante do cuidado e gerenciamento da situação:

"Nunca tínhamos passado por isso, foi um baque, usar bolsa de colostomia, o que é isso? [...] Um dia eu cheguei em casa chorando, meu Deus do céu eu não vou saber cuidar desse piá, não vou conseguir [...].” (P3)

"Eu não conseguia passar a mão na colostomia, eu tinha medo de machucar [...], levei ele pra casa e não sabia dar banho, foi um dos meus problemas, não sabia dar banho numa criança com uma ferida aberta na barriga.” (P6)

"Nunca tivemos apoio psicológico aqui, acho que foi o que faltou pra gente, como era uma coisa nova, não sabíamos como lidar e o que esperar." (P9)

Percebe-se, ainda, durante a escuta aos pais, a falta de segurança, de conversa e de troca com a equipe multiprofissional, gerando sentimentos de angústia e abandono, quando o relatado é a falta de alguém que entenda, oriente e dê suporte. Vemos que, além de muito cansativo, o desejo dos pais é que o filho receba tratamento e volte logo para casa e para o convívio familiar, pois lá terão mais compreensão ${ }^{12}$.

A falta de recursos e formação da equipe profissional, em conjunto com a falta de informações recebidas, culmina não só em sentimentos de medo, incerteza e desamparo, como também traz problemas à criança com estomia ${ }^{10}$.

No estado do Rio Grande do Sul (RS), participantes de um grupo de apoio à pessoa com estomia citam a falta de sistematização do cuidado e orientações para a alta hospitalar e percebem que há insuficiência de conhecimentos específicos sobre cuidado com as estomias intestinais por parte de profissionais, o que dificulta o processo de aprendizagem e posterga a adaptação à nova realidade ${ }^{13}$.

\section{O papel da rede de apoio no cuidado domiciliar da criança com estomia}

Os participantes demonstraram, em suas falas, que, diante da falta de informação a respeito de como cuidar da estomia de seus filhos, foram levados a buscar por fontes alternativas de informação, como internet, pais de outras crianças, associações e enfermeiros de empresas, conforme demonstrado nos trechos a seguir:

"A mãe de um paciente me ajudou depois que fui para casa, muitas coisas ela foi me falando como fazia e era melhor.”(P2)

“[...] eu fui na associação, eles deram uma aula para gente e explicaram direitinho, lá eles têm mais tempo, aqui eles não têm muito tempo." (P3) 
"A gente até tem seguido algumas coisas de colostomia no

Facebook, tem bastante coisa pra gente tirar dúvida [...].”(P9)

A busca por fontes alternativas contribuiu para os pais reduzirem a ansiedade gerada por não saber prestar o cuidado e para permitir a convivência com pessoas que passaram por situações semelhantes.

Destaca-se que as relações sociais exercem papel relevante na vida da pessoa com estomia. $\mathrm{O}$ apoio social é um processo que envolve relações e facilita a moderação e o equilíbrio em situações de estresse e ansiedade. Estudo realizado em relação ao apoio social recebido constatou que o apoio social teria sido "suficiente" em 49,5\% dos casos e "insuficiente" em outros $36,5 \%$, e que não houve associação do resultado com o perfil socioeconômico ${ }^{14}$.

Os resultados referentes à busca por redes de apoio e estratégias alternativas de aprendizagem diante da falha das orientações para alta hospitalar também foram encontrados em estudo realizado em um grupo de apoio à pessoa com estomia com participantes adultos que relataram a inexistência de orientações, prolongando o processo de aprendizagem e levando à necessidade de busca por outras maneiras de suprir as demandas da estomização. Esses participantes citam terem aprendido lendo orientações de uso do equipamento coletor na embalagem, procurando na internet ou sozinhos, na prática ${ }^{13}$

Em contrapartida, não se pode deixar de ressaltar que existe um risco relacionado à falta de cientificidade de algumas fontes citadas pelos participantes que pode levar a condutas inadequadas que venham resultar em complicações à criança.

Diante do exposto, pode-se apreender um efeito cíclico de complicações geradas pela falha nas orientações de cuidado à criança com estomia durante a hospitalização. Com a falta de orientação, os pais expõem as crianças a condutas inadequadas que levam ao aparecimento de complicações. Essas, consequentemente, tendem a intensificar a insegurança e levar os pais a procurar fontes alternativas de orientação.

\section{Conhecimento gera segurança para cuidar da criança com estomia}

Alguns participantes referiram acreditar que ter recebido mais informações sobre o cuidado com a estomia poderia ter evitado sofrimento e permitido que tivessem mais tranquilidade e segurança para cuidar dos seus filhos.

"Se tivéssemos mais informação não passaríamos por tudo isso." (P3)
“Tudo que é a primeira vez, primeira situação, é um impacto para todos; depois que você começa a lidar com a colostomia se torna mais fácil.” (P4)

"Na verdade foram boas as orientações deles, o que eles nos ensinaram. Nós não sabíamos nada sobre colostomia.”(P7)

Foi possivel observar que os pais entrevistados consideram que o conhecimento e a experiência adquiridos, por meios alternativos ou pela própria vivência do cuidado, tendem a amenizar sentimentos negativos como insegurança, medo e impotência.

A literatura atual reforça os achados do estudo, citando que a orientação técnica dos cuidados com a estomia tem a capacidade de reduzir os sentimentos negativos, porém ainda há necessidade da inclusão dos aspectos emocionais, sociais, culturais e espirituais na assistência perioperatória, que deve contemplar acolhimento, além do ensino sobre a cirurgia e suas consequências, envolvendo familiares e possibilitando participação efetiva ${ }^{11}$.

\section{Estratégias que poderiam contribuir para o cuidado da criança com estomia no domicílio}

A amostra estudada foi questionada quanto à forma que gostariam de ter recebido as orientações a respeito do cuidado de seus filhos. Observou-se, nas falas, a riqueza de conteúdo nas sugestões a respeito de estratégias que o serviço poderia adotar para melhor condução dessas orientações. Dentre as possibilidades, foram citados livros, fôlderes e vídeos ilustrativos, bem como profissionais especializados para repassar as orientações necessárias:

"Eu acho que num livrinho, porque pelo menos você levaria para casa e qualquer dúvida que você tivesse iria procurar." (P1)

"Podia ter vídeos explicando como cuidar ou um livro mostrando os cuidados com o estoma. Aqui no hospital tinha que ter uma pessoa especializada para estoma, porque tem muitos enfermeiros que nem sabem o que é estoma e como cuidar, $[\ldots]$ orientar melhor a gente e alguma coisa que possa levar para casa.” (P2)

"Eu acho que deveria ter um espaço para essas mães porque só quem passou que sabe como vai ajudar outra mãe.”(P6) 
Observou-se que a maioria dos participantes sugeriu um guia impresso, seja no formato de fôlder ou manual, demonstrando a carência que sentiram de material de apoio confiável para tirar suas dúvidas diante da dificuldade no cuidado.

A maioria dos materiais didáticos atualmente disponíveis descreve cuidados com colostomia para adultos, sendo poucos os que se dirigem às necessidades das crianças e de seus pais. Estudo realizado na Índia em rede de nível terciário com uso de auxílios educacionais audiovisuais no ensino de pais de crianças com colostomia gerou o aumento nos índices de conhecimento imediatamente após a técnica realizada. Mostrou, ainda, melhora significativa nos escores de conhecimento e habilidades com as orientações fornecidas em ambiente hospitalar ${ }^{15}$.

Pacientes avaliados em outros estudos acreditam que o sistema de saúde deve ser mais focado na formação de enfermeiros estomaterapeutas, considerando-os referência no processo de atenção à saúde de pessoas com estomia. Os entrevistados indicaram, ainda, como necessidades de melhorias para o sistema, a inserção da especialidade em todas as fases dos cuidados de saúde, especialmente no âmbito dos centros de saúde pública, onde as pessoas com estomia recebem os primeiros cuidados pós-alta hospitalar ${ }^{10}$.

Estudo realizado em Santa Maria (RS), com objetivo de conhecer as percepções de participantes de um grupo de apoio para pessoas com colostomia sobre a utilização de um vídeo educativo, obteve como resultado que a tecnologia apresentada foi considerada válida enquanto estratégia complementar para educação em saúde dessas pessoas e seus familiares. Um dos participantes do estudo citado relata que se o vídeo apresentado na associação o fosse ainda no hospital onde a estomia foi construída, eles já sairiam mais fortalecidos ${ }^{13}$. Essa pesquisa demonstra coerência e aplicabilidade da sugestão de utilização do vídeo no processo de capacitação para cuidado com a estomia, apresentada por duas famílias participantes.

Vale citar que, durante a elaboração deste artigo, a instituição onde a coleta de dados foi realizada já se encontrava em fase de remodelação da assistência prestada a essa população, em resposta aos resultados apresentados. Um manual de orientações para pais de crianças com estomia intestinal está em fase de elaboração, com base nas sugestões dos participantes e em literatura atual. Além disso, o atendimento pré- e pós-operatório começa a ser realizado pelos enfermeiros especialistas da instituição em parceria com a equipe multiprofissional.

\section{CONCLUSÃO}

Identificou-se, durante a realização do estudo, uma falha considerável na orientação e apoio aos pais de crianças com estomia na instituição estudada. As consequências dessas lacunas do conhecimento são as complicações relacionadas a condutas equivocadas, sentimentos de medo e incapacidade dos pais para o cuidado da criança.

A rede de apoio atuou de forma positiva e negativa, tendo em vista os equívocos e as lacunas de conhecimento para o cuidado domiciliar da criança com estomia, pois muitas vezes os pais utilizaram ferramentas populares sem fundamento teórico.

O tamanho da amostra foi limitado, porém determinado pelo número de cirurgias que ocorreram nesse triênio, levando em consideração os critérios de inclusão, como o cuidado domiciliar da criança com estomia e o atual acompanhamento ambulatorial na instituição, o que acabou por limitar a análise quantitativa e impediu tratamento estatístico, sendo apresentado apenas estatística descritiva, tornando a associação entre as variáveis inviável.

O estudo demonstrou, também, a importância dos feedbacks para auxílio nas mudanças e sugestões de estratégias válidas que poderão contribuir para implementação de um fluxo de atendimento e orientações para o cuidado de crianças com estomia.

Tendo em vista a responsabilidade das instituições hospitalares e dos profissionais quanto às orientações para alta da criança com estomia, faz-se necessária a assunção dos papéis da rede como um todo, dando suporte à família, peça importante na relação direta ao processo de cuidar, de modo a torná-la apta e capaz de exercer todas as atividades relacionadas ao cuidado direto a essa criança, permitindo melhorias na sua $Q V$ e também da família e comunidade na qual está inserida.

Com relação às limitações desta pesquisa, foi possível perceber que a literatura que aborda cuidados diretos com a estomia e orientações necessárias à alta hospitalar em pediatria é bastante escassa, o que nos traz a necessidade de ampliar os estudos nessa área.

Para o futuro, sugerem-se a realização de estudos multicêntricos com amostras maiores e pesquisas prospectivas que testem a efetividade de métodos 


\section{CONTRIBUIÇÃO DOS AUTORES}

sistemáticos e distintos de orientação na redução das complicações das estomias e periestomas das crianças, bem como a atenuação dos sentimentos de angústia, medo e insegurança dos pais no cuidado dessas crianças.
Conceitualização, Maia EMB e Assis GM; Metodologia, Maia EMB e Assis GM; Investigação, Maia EMB; Redação - Primeira versão, Maia EMB; Redação - Revisão \& Edição, Maia EMB e Assis GM; Supervisão, Assis GM.

\section{REFERÊNCIAS}

1. Melo MC, Kamada I. O papel da família no cuidado à criança com estoma intestinal: uma revisão narrativa. ESTIMA, Braz J Enterostomal Ther. 2015;13(3):43-9.

2. Paula MAB, Paula PR, Cesaretti IUR, organizadores. Estomaterapia em foco e o cuidado especializado. São Caetano do Sul: Yendis; 2014.

3. Zacarin CFL, Alvarenga WA, Souza ROD, Borges DCS, Dupas G. Vulnerabilidade da família de crianças com estomia intestinal. Revista Eletrônica de Enfermagem. 2014;16(2):426-33. https://doi.org/ 0.5216/ree.v16i2.26639

4. Monteiro SNC, Melo MC, Kamada I, Silva AL. Caracterização de cuidadores de crianças e adolescentes estomizados atendidos em serviço de reabilitação. ESTIMA, Braz J Enterostomal Ther. 2016;14(2):76-83. https://doi. org/10.5327/z1806-3144201600020005

5. Diniz IV, Matos SDO, Brito KKG, Andrade SSC, Oliveira SHS, Oliveira MJGO. Nursing care applied to children with an ostomy arising from Hirschsprung's disease. Rev enferm UFPE on line. 2016; 10(3): 1119-26. https://doi.org/10.5205/ reuol.8702-76273-4-SM.1003201623

6. Almeida EJ de, Silva AL da. Caracterização do perfil epidemiológico dos estomizados em hospitais da Secretaria de Estado de Saúde do Distrito Federal. ESTIMA, Braz J Enterostomal Ther. 2015;13(1):11-6. https://doi. org/10.5327/Z1806-3144201500010004

7. Bardin L. Análise de conteúdo. Trad. Reta LA, Pinheiro A. São Paulo: Edições 70; 2016.

8. Costa ECL da, Vale DS do, Luz MHBA. Perfi das crianças estomizadas em um hospital público de Teresina, Piauí. ESTIMA, Braz J Enterostomal Ther. 2016;14(4):169-74. https://doi.org/10.5327/Z18063144201600040003
9. Silva JC, Borsatto AZ, Teixeira ER, Umpiérrez AF. Demarcação abdominal por enfermeira estomoterapêuta. Enfermería (Montev.). 2017;6(1):12-8. https://doi.org/10.22235/ech. $\underline{\text { v6i1.1365 }}$

10. Nieves CB de las, Díaz CC, Celdrán-Mañas M, MoralesAsencio JM, Hernández-Zambrano SM, Hueso-Montoro C. Percepção de pacientes ostomizados sobre os cuidados de saúde recebidos. Rev Latino-Am Enfermagem. 2017;25: e2961. https://doi.org/10.1590/1518-8345.2059.2961

11. Silva NM, Santos MA dos, Rosado SR, Galvão CM, Sonobe HM. Aspectos psicológicos de pacientes estomizados intestinais: revisão integrativa. Rev Latino-Am Enfermagem. 2017;25:e2950. $\underline{8345.2231 .2950}$

https://doi.org/10.1590/1518-

12. Sélos PR, Costa PCP, Toledo VP. Vivendo em casa de apoio durante o tratamento do câncer infantil: percepções maternas. Rev enferm UFPE on line. 2014;8(6):1474-81.

13. Dalmolin A, Girardon-Perlini NMO, Coppetti LC, Rossato GC, Gomes JS, Silva MEN. Vídeo educativo como recurso para educação em saúde a pessoas com colostomia e familiares. Rev Gaúcha Enferm. 2016;37(Espec):e68373. https://doi. org/10.1590/1983-1447.2016.esp.68373

14. Reis FF dos, Carvalho AAS, Santos CSB, Rodrigues VMCP. Percepção sobre o apoio social do homem colostomizado na região norte de Portugal. Esc Anna Nery. 2014;18(4):5707. https://doi.org/10.5935/1414-8145.20140081

15. Dabas H, Sharma KK, Joshi P, Agarwala S. Video teaching program on management of colostomy: Evaluation of its impact on caregivers. I Indian Assoc Pediatr Surg. 2016;21(2):54-6. ht htps://doi.org/10.4103/0971$\underline{9261.176933}$ 